\title{
La hernia paraduodenal como reto diagnóstico-terapéutico y su abordaje por mínima invasión
}

\section{Paraduodenal hernia as a diagnostic-therapeutic challenge and its minimally invasive approach}

\author{
Rodolfo Chávez-Magallón, * José Luis Beristain-Hernández* \\ * Departamento de Cirugía General. Hospital de Especialidades «Dr. Antonio Fraga Mouret» \\ del Centro Médico Nacional La Raza del Instituto Mexicano del Seguro Social.
}

\section{RESUMEN}

Introducción: Las hernias internas congénitas son una condición poco común y representan menos de $1 \%$ de las hernias abdominales. La oclusión intestinal por una hernia interna ocurre en $0.25-0.9 \%$. La hernia paraduodenal o hernia de Treitz es la hernia interna más común (53\%). Deben ser consideradas como posibilidad diagnóstica siempre que se presente un paciente con oclusión intestinal sin antecedente de cirugía abdominal y en el que se hayan descartado otras causas. Presentación del caso: Hombre de 61 años de edad, quien se presenta con dolor abdominal crónico, sin antecedentes de cirugía abdominal. Posterior a protocolo de estudio de laboratorio, en la tomografía axial computada se encuentra una imagen sugestiva de hernia paraduodenal izquierda. Se realiza laparoscopía diagnóstica, donde se encontró una hernia interna paraduodenal izquierda, misma que se libera y reduce sin complicaciones. El paciente es egresado a los tres días de posoperado, asintomático. Conclusiones: La hernia paraduodenal fue descrita inicialmente por Neubauer en 1786, la teoría más aceptada sobre la fisiopatología fue descrita por Andrews en 1923, sugiriendo un error embriológico durante la rotación del intestino medio primitivo sobre la arteria mesentérica superior. Se presentan con mayor frecuencia en hombres y en el lado izquierdo, y al menos $50 \%$ de los pacientes con hernia paraduodenal desarrollará oclusión intestinal.

Palabras clave: Hernia paraduodenal, hernia interna congénita, hernia de Treitz.

\section{ABSTRACT}

Introduction: Congenital internal hernias are a rare condition, accounting for less than $1 \%$ of abdominal hernias. Intestinal obstruction due to an internal hernia occurs in $0.25-0.9 \%$. Paraduodenal hernia also called Treitz hernia is the most common internal hernia (53\%). Diagnosis should be considered in any patient with intestinal obstruction with no previous history of abdominal surgery and in whom other causes have been ruled out. Case presentation: We present a case of a 61-yearold male, who presented with an acute abdominal condition due to an intestinal obstruction with no history of abdominal surgery. An emergency laparoscopic surgery was performed, with the finding of a left paraduodenal internal hernia, which is reduced without complications and the patient was discharged home asymptomatic on the 3rd post-operative day. Conclusions: Paraduodenal hernia was initially described by Neubauer in 1786, the most accepted theory about the pathophysiology was described by Andrews in 1923, who suggests an embryological error during the rotation of the primitive midgut around the superior mesenteric artery. The incidence is higher in men and on the left side and at least 50\% of patients with paraduodenal hernia will develop intestinal occlusion.

Keywords: Paraduodenal hernia, congenital internal hernia, Treitz hernia. 


\section{INTRODUCCIÓN}

La hernia interna es una protrusión anormal de una víscera (comúnmente intestino delgado) a través de un defecto, que puede ser congénito o adquirido. ${ }^{1}$ Es una patología relativamente rara, producto de la herniación del intestino a través de un defecto en el peritoneo o mesenterio, resultado de un defecto en la rotación del intestino medio. ${ }^{2}$

Las hernias internas son una condición poco común, representan menos de $1 \%$ de las hernias abdominales. ${ }^{1}$ La oclusión intestinal debida a una hernia interna ocurre en $0.25-0.9 \%$ aproximadamente. ${ }^{3}$ Pueden clasificarse de acuerdo con su ubicación; las más comunes son las hernias paraduodenales, que representan más de $50 \%$ de las hernias internas, seguida de la pericecal (13\%), del hiato de Winslow $(8 \%)$, transmesentérica $(8 \%)$, del mesocolon sigmoides $(6 \%)$, pélvica o supravesical $(6 \%)$ y transomental (1-4\%). ${ }^{4,5}$

La hernia paraduodenal es una hernia interna que se forma cerca del ligamento de Treitz, ${ }^{6}$ por lo que también es conocida como hernia de Treitz; ${ }^{2}$ debe ser considerada como posibilidad diagnóstica siempre que se presente un paciente con oclusión intestinal sin antecedentes quirúrgicos abdominales. ${ }^{7}$ Las hernias paraduodenales pueden ser clasificadas a su vez en derecha e izquierda, siendo la izquierda la más común $(75 \%){ }^{3}$

\section{PRESENTACIÓN DEL CASO}

Hombre de 61 años de edad, sin antecedentes quirúrgicos abdominales, con historia de dolor abdominal de un año de evolución, el cual se presenta de manera intermitente, localizado en hemiabdomen izquierdo; ha recibido manejo con analgésicos, antiespasmódicos y otros medicamentos sin mejoría significativa.

Acude de forma espontánea al Servicio de Urgencias, con dolor abdominal en hemiabdomen izquierdo, en esta ocasión de 72 horas de evolución, relacionado con la ingesta de alimentos; niega otra sintomatología.

El estudio de laboratorio realizado al ingreso mostró lo siguientes resultados: glucosa $89 \mathrm{mg} / \mathrm{dL}$, creatinina sérica $1 \mathrm{mg} / \mathrm{dL}$, sodio $141 \mathrm{mmol} / \mathrm{L}$, potasio $4.4 \mathrm{mmol} / \mathrm{L}$, TGP 18 U/L, TGO $18 \mathrm{U} / \mathrm{L}$, DHL $320 \mathrm{U} / \mathrm{L}$, amilasa $72 \mathrm{U} / \mathrm{L}$, bilirrubina total $0.37 \mathrm{mg} / \mathrm{dL}$, hemoglobina $15.7 \mathrm{~g} / \mathrm{dL}$, leucocitos $\mathrm{K} / \mu \mathrm{L}$, neutrófilos $41.4 \%$, plaquetas $169,000 / \mu \mathrm{L}$.

Se realiza estudio de imagen tomografía axial computarizada con doble contraste (oral e intravenoso), donde se observa conglomerado de asas de yeyuno en cuadrante superior izquierdo, cerca del ligamento de Treitz, sin paso del medio de contraste hacia el íleon, sugerente de hernia interna (Figura 1).

Ante la presencia de un paciente con dolor abdominal crónico, con datos sugestivos de hernia interna, se decide realizar laparoscopía diagnóstica de forma urgente. Con la colocación de tres trocares, el primero de $12 \mathrm{~mm}$ a $1 \mathrm{~cm}$ por arriba de la cicatriz umbilical, el segundo y tercero en el flanco derecho e izquierdo, respectivamente, ambos de 5 mm (Figura 2). Se Inicia el abordaje con la identificación del ligamento de Treitz (Figura 3); se continuó con la exploración del intestino delgado hasta identificar la hernia interna paraduodenal izquierda en el sitio de oclusión (Figura 4), la cual se encontró sin cambios isquémicos intestinales, por lo que se libera cortando con electrocoagulación, reduciendo la hernia sin complicaciones (Figura 5).

El paciente presentó evolución posquirúrgica favorable y fue egresado a los tres días de posoperado, asintomático. Se mantuvo en seguimiento durante un año sin presentar recidiva por lo que se dio de alta definitiva.
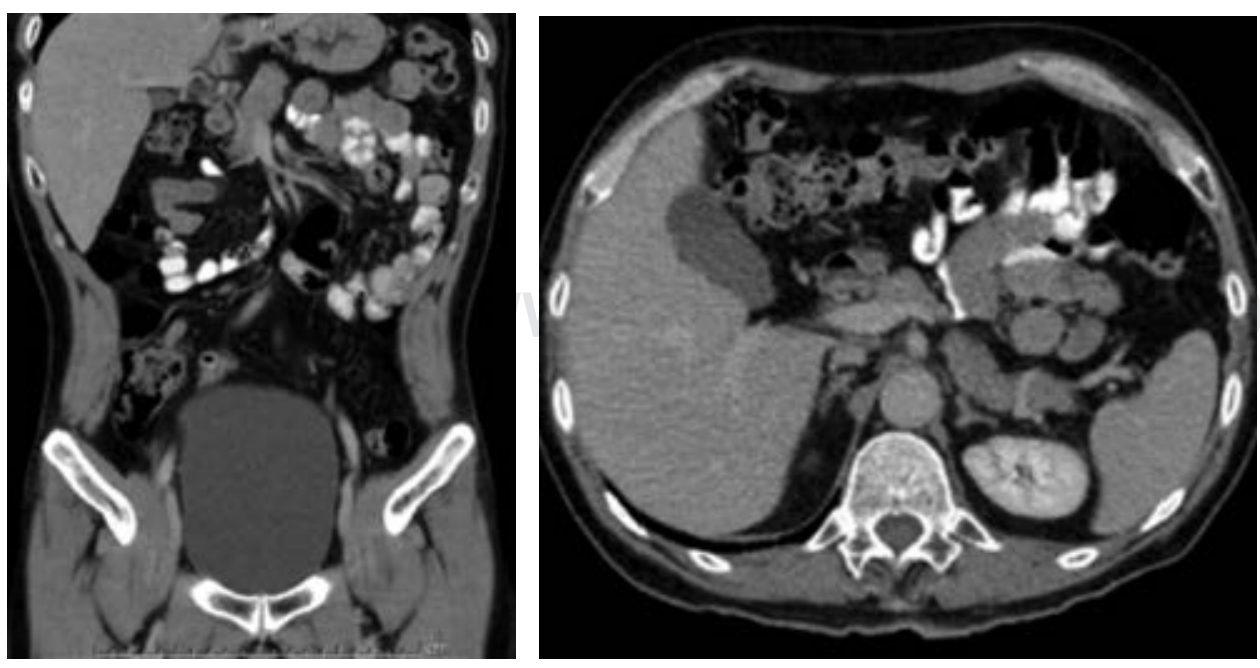

Figura 1:

Tomografía computada abdominal. Se observa conglomerado de asas de yeyuno en cuadrante superior izquierdo, cerca del ligamento de Treitz, sin paso del medio de contraste hacia el íleon, sugerente de hernia interna. 
Figura 2:

Esquema colocación de puertos para abordaje laparoscópico. Primer trocar: trocar de $10 \mathrm{~mm}$ en línea media, a $1 \mathrm{~cm}$ por arriba de

la cicatriz umbilical, para lente de la cámara. Segundo y tercer trocares de $5 \mathrm{~mm}$ en línea media clavicular derecha e izquierda, a $3 \mathrm{~cm}$ por cal, para la instrumentación, tracción y disección debajo de la cicatriz umbili-

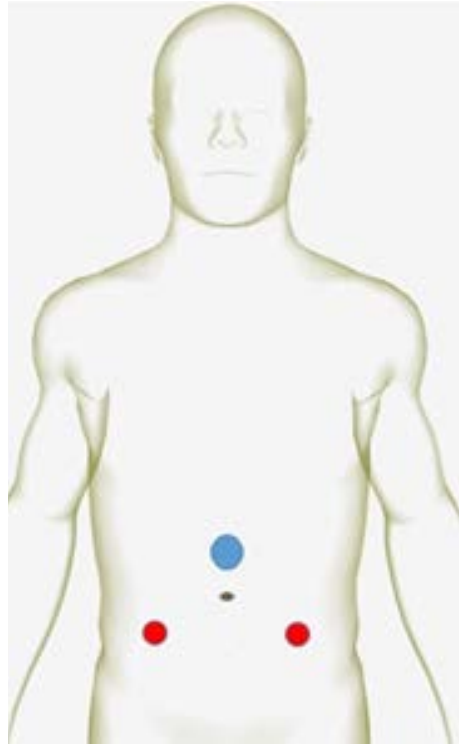

\section{DISCUSIÓN}

La hernia paraduodenal fue descrita inicialmente por $\mathrm{Ne}$ ubauer en 1786 y clasificada por Treitz en 1827. ${ }^{8}$ Existen múltiples teorías sobre la fisiopatología en la formación de la hernia interna; la más aceptada fue descrita por Andrews en 1923, quien sugiere un error embriológico durante la rotación del intestino medio primitivo. ${ }^{1}$ La arteria mesentérica superior constituye el eje de esta rotación e irriga el intestino medio.

La hernia paraduodenal izquierda involucra la fosa de Landzert, la cual está localizada lateral a la cuarta porción del duodeno y posterior a la vena mesentérica inferior y a la arteria cólica izquierda. ${ }^{3}$ Se presenta con mayor frecuen-

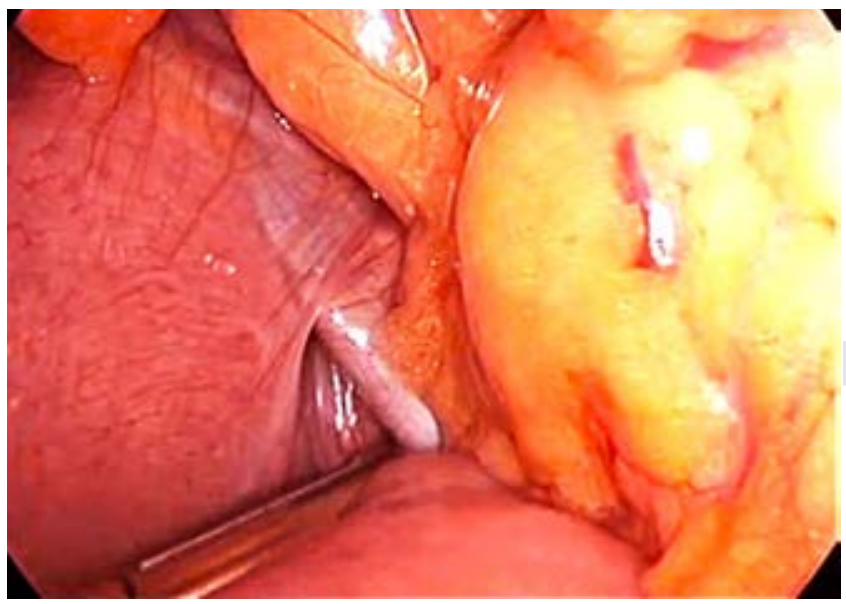

Figura 3: Inicio de la exploración laparoscópica a nivel del ligamento de Treitz; se aprecia la vena mesentérica inferior.

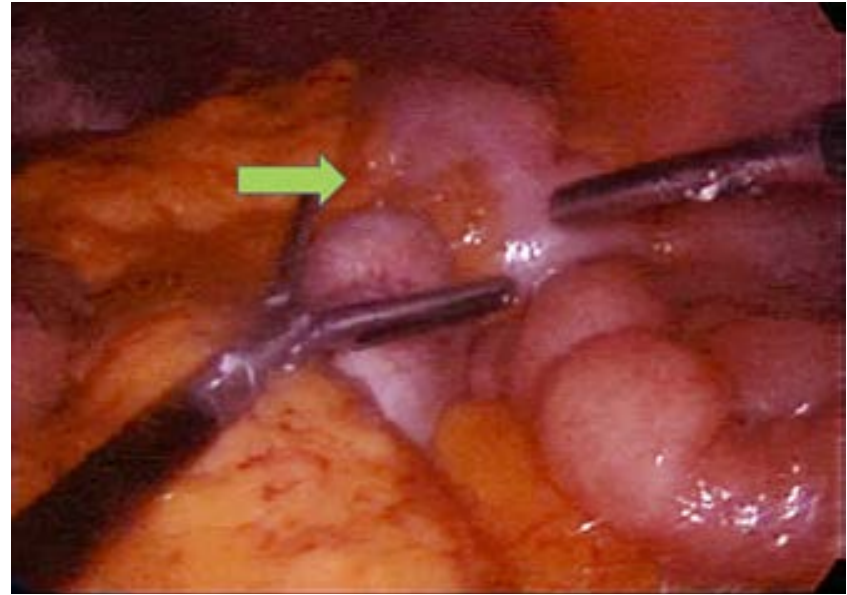

Figura 4: Hernia interna. Sitio de oclusión intestinal, se observa transición de asas señalado con flecha.

cia en hombres y en el lado izquierdo, en una proporción 3:1 en comparación con la hernia paraduodenal derecha, también conocida como hernia de Waldeyer. ${ }^{1,8}$

Al menos $50 \%$ de los pacientes con hernia paraduodenal desarrollarán oclusión intestinal, por lo que se recomienda la cirugía en todos los casos. ${ }^{6}$ Algunos casos pueden presentar sintomatología desde los primeros años de vida, en nuestro paciente ésta inició de manera tardía a los 60 años de edad.

\section{CONCLUSIONES}

Debemos tener siempre en cuenta el diagnóstico en pacientes con oclusión intestinal, así como alto nivel de sos-

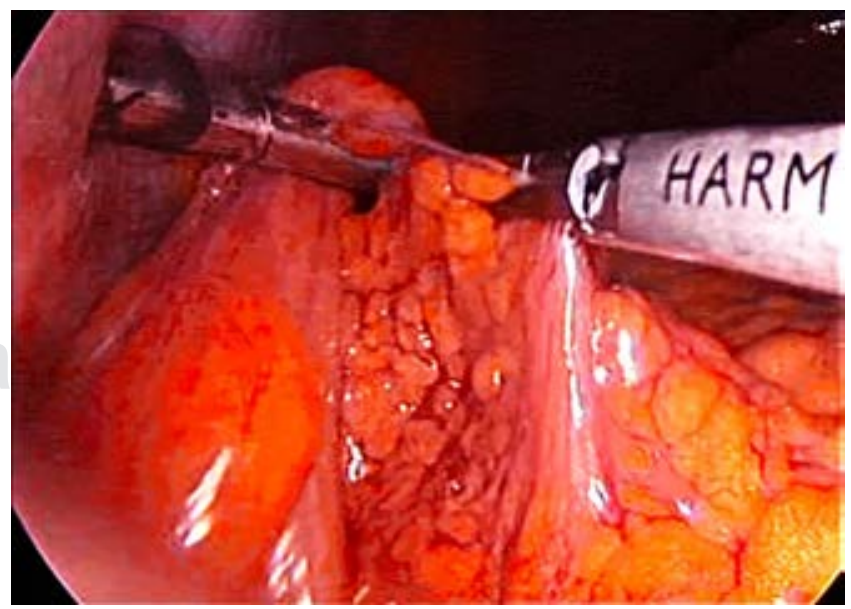

Figura 5: Liberación de la hernia interna con coagulación bipolar avanzada. 
pecha en casos sin antecedentes quirúrgicos abdominales. Es importante identificar y buscar de forma intencionada defectos de la rotación del intestino al realizar una laparoscopía diagnóstica, ya que pueden pasar desapercibidos durante la cirugía.

En casos de abordaje laparoscópico en oclusión intestinal o de pacientes con hernias internas, siempre se sugiere realizar el abordaje mediante técnica de Hasson, colocando el primer trocar en línea media, a $1 \mathrm{~cm}$ por arriba de la cicatriz umbilical, introduciendo lente de la cámara para verificar posición adecuada, y posteriormente la inducción del neumoperitoneo a 10 a $12 \mathrm{mmHg}$ con dióxido de carbono a una velocidad de $12 \mathrm{mmHg} / \mathrm{min}$.

Se sugiere la colocación de dos trocares adicionales, de $5 \mathrm{~mm}$ en línea media clavicular derecha e izquierda, $3 \mathrm{~cm}$ por debajo de la cicatriz umbilical, mismos que servirán para la instrumentación, tracción y disección.

Se procede a realizar la revisión de la cavidad abdominal, realizando con una pinza intestinal corta la tracción del colon transverso con dirección cefálica, a fin de identificar la emergencia del yeyuno y, a partir del ligamento de Treitz, se inicia la exploración del intestino delgado hasta identificar el sitio de oclusión para liberarlo y, en caso necesario, realizar resección intestinal.

\section{REFERENCIAS}

1. Kadhem S, Ali MH, Al-Dera FH, Alzayer NA, Alyagoub HM. Left paraduodenal hernia: case report of rare cause of recurrent abdominal pain. Cureus. 2020; 12: e7156.

2. Kabbani D, Salem A, Holloway DK. Paraduodenal herniation: an internal herniation in a virgin abdomen. Int J Surg Case Rep. 2014; 5: 1148-1150.

3. Kulkarni GV, Salgaonkar HP, Sharma PC, Chakkarvarty NR, Katara AN, Bhandarkar DS. Laparoscopic repair of left paraduodenal hernia: Report of two cases and review of the literature. Asian J Endosc Surg. 2016; 9: 157-160.

4. Husain A, Bhat S, Roy AK, Sharma V, Dubey SA, Faridi MS. Internal hernia through paraduodenal recess with acute intestinal obstruction: a case report. Indian J Surg. 2012; 74: 354-355.

5. Sakamoto T, Lefor AK. Laparoscopic reduction and repair of a left paraduodenal hernia. BMJ Case Rep. 2019; 12 : e232098.

6. Wakabayashi M, Kono S, Takahashi T. Laparoscopic repair of acute small bowel obstruction due to left paraduodenal hernia: A case report. Int J Surg Case Rep. 2018; 51: 194-199.

7. Hussein M, Khreiss M, Al-Helou G, Alaeddine M, Elias E, Abi Saad GS. Laparoscopic repair of a left paraduodenal hernia presenting with acute bowel obstruction: report of a case. Surg Laparosc Endosc Percutan Tech. 2012; 22: e28-e30.

8. Morán JM, Salas J, Sanjuán S, González G, Gallello A, Serrano A et al. Incarceración retroperitoneal del yeyuno en una hernia paramesocólica izquierda. Cir Pediatr. 2002; 15: 168-171. 